\title{
SemEval-2020 Task 12: Multilingual Offensive Language Identification in Social Media (OffensEval 2020)
}

\author{
Marcos Zampieri ${ }^{1}$, Preslav Nakov ${ }^{2}$, Sara Rosenthal $^{3}$, Pepa Atanasova ${ }^{4}$, Georgi Karadzhov ${ }^{5}$ \\ Hamdy Mubarak ${ }^{2}$, Leon Derczynski ${ }^{6}$, Zeses Pitenis ${ }^{7}$, Çă̆rı Çöltekin ${ }^{8}$ \\ ${ }^{1}$ Rochester Institute of Technology, USA, ${ }^{2}$ Qatar Computing Research Institute, Qatar \\ ${ }^{3}$ IBM Research, USA, ${ }^{4}$ University of Copenhagen, Denmark, ${ }^{5}$ University of Cambridge, UK \\ ${ }^{6}$ IT University Copenhagen, Denmark, ${ }^{7}$ University of Wolverhampton, UK \\ ${ }^{8}$ University of Tübingen, Germany \\ marcos.zampierierit.edu
}

\begin{abstract}
We present the results and the main findings of SemEval-2020 Task 12 on Multilingual Offensive Language Identification in Social Media (OffensEval-2020). The task included three subtasks corresponding to the hierarchical taxonomy of the OLID schema from OffensEval-2019, and it was offered in five languages: Arabic, Danish, English, Greek, and Turkish. OffensEval-2020 was one of the most popular tasks at SemEval-2020, attracting a large number of participants across all subtasks and languages: a total of 528 teams signed up to participate in the task, 145 teams submitted official runs on the test data, and 70 teams submitted system description papers.
\end{abstract}

\section{Introduction}

Offensive language is ubiquitous in social media platforms such as Facebook, Twitter, and Reddit, and it comes in many forms. Given the multitude of terms and definitions related to offensive language used in the literature, several recent studies have investigated the common aspects of different abusive language detection tasks (Waseem et al., 2017; Wiegand et al., 2018). One such example is SemEval-2019 Task 6: OffensEval (Zampieri et al., 2019b), which is the precursor to the present shared task. OffensEval2019 used the Offensive Language Identification Dataset (OLID), which contains over 14,000 English tweets annotated using a hierarchical three-level annotation schema that takes both the target and the type of offensive content into account (Zampieri et al., 2019a). The assumption behind this annotation schema is that the target of offensive messages is an important variable that allows us to discriminate between, e.g., hate speech, which often consists of insults targeted toward a group, and cyberbullying, which typically targets individuals. A number of recently organized related shared tasks followed similar hierarchical models. Examples include HASOC-2019 (Mandl et al., 2019) for English, German, and Hindi, HatEval-2019 (Basile et al., 2019) for English and Spanish, GermEval-2019 for German (Struß et al., 2019), and TRAC-2020 (Kumar et al., 2020) for English, Bengali, and Hindi.

OffensEval-2019 attracted nearly 800 team registrations and received 115 official submissions, which demonstrates the interest of the research community in this topic. Therefore, we organized a follow-up, OffensEval-2020 (SemEval-2020 Task 12), which is described in this report, building on the success of OffensEval-2019 with several improvements. In particular, we used the same three-level taxonomy to annotate new datasets in five languages, where each level in this taxonomy corresponds to a subtask in the competition:

- Subtask A: Offensive language identification;

- Subtask B: Automatic categorization of offense types;

- Subtask C: Offense target identification.

This work is licensed under a Creative Commons Attribution 4.0 International License. License details: http: //creativecommons.org/licenses/by/4.0/.

${ }^{1}$ http://sites.google.com/site/offensevalsharedtask/offenseval2019

${ }^{2}$ http://sites.google.com/site/offensevalsharedtask/home 
The contributions of OffensEval-2020 can be summarized as follows:

- We provided the participants with a new, large-scale semi-supervised training dataset containing over nine million English tweets (Rosenthal et al., 2020).

- We introduced multilingual datasets, and we expanded the task to four new languages: Arabic (Mubarak et al., 2020b), Danish (Sigurbergsson and Derczynski, 2020), Greek (Pitenis et al., 2020), and Turkish (Çöltekin, 2020). This opens the possibility for cross-lingual training and analysis, which several participants indeed explored.

- Compared to OffensEval-2019, we used larger test datasets for all subtasks.

Overall, OffensEval-2020 was a very successful task. The huge interest demonstrated last year continued this year, with 528 teams signing up to participate in the task, and 145 of them submitting official runs on the test dataset. Furthermore, OffensEval-2020 received 70 system description papers, which is an all-time record for a SemEval task.

The remainder of this paper is organized as follows: Section 2 describes the annotation schema. Section 3 presents the five datasets that we used in the competition. Sections 4-9 present the results and discuss the approaches taken by the participating systems for each of the five languages. Finally, Section 10 concludes and suggests some possible directions for future work.

\section{Annotation Schema}

OLID's annotation schema proposes a hierarchical modeling of offensive language. It classifies each example using the following three-level hierarchy:

\section{Level A - Offensive Language Detection}

Is the text offensive (OFF) or not offesive (NOT)?

NOT: text that is neither offensive, nor profane;

OFF: text containing inappropriate language, insults, or threats.

\section{Level B - Categorization of Offensive Language}

Is the offensive text targeted (TIN) or untargeted (UNT)?

TIN: targeted insults or threats towards a group or an individual;

UNT: untargeted profanity or swearing.

\section{Level C - Offensive Language Target Identification}

Who or what is the target of the offensive content?

IND: the target is an individual, which can be explicitly mentioned or it can be implicit;

GRP: the target is a group of people based on ethnicity, gender, sexual orientation, religious belief, or other common characteristic;

OTH: the target does not fall into any of the previous categories, e.g., organizations, events, and issues.

\section{Data}

In this section, we describe the datasets for all five languages: Arabic, Danish, English, Greek, and Turkish. All of the languages follow the OLID annotation schema and all datasets were pre-processed in the same way, e.g., all user mentions were substituted by QUSER for anonymization. The introduction of new languages using a standardized schema with the purpose of detecting offensive and targeted speech should improve dataset consistency. This strategy is in line with current best practices in abusive language data collection (Vidgen and Derczynski, 2020). All languages contain data for subtask A, and only English contains data for subtasks B and C. The distribution of the data across categories for all languages for subtask A is shown in Table 1, while Tables 2 and 3 present statistics about the data for the English subtasks B and C, respectively. Labeled examples from the different datasets are shown in Table 4. 


\begin{tabular}{lrrrrrrrr}
\hline & \multicolumn{3}{c}{ Training } & & \multicolumn{3}{c}{ Test } \\
\cline { 2 - 3 } \cline { 8 - 9 } Language & \multicolumn{1}{c}{ OFF } & \multicolumn{1}{c}{ NOT } & Total & & OFF & NOT & Total \\
\hline English & 1448861 & 7640279 & 089140 & & 1090 & 2807 & 3897 \\
Arabic & 1589 & 6411 & 8000 & & 402 & 1598 & 2000 \\
Danish & 384 & 2577 & 2961 & & 41 & 288 & 329 \\
Greek & 2486 & 6257 & 8743 & & 425 & 1119 & 1544 \\
Turkish & 6131 & 25625 & 31756 & & 716 & 2812 & 3528 \\
\hline
\end{tabular}

Table 1: Subtask A (all languages): statistics about the data.

\begin{tabular}{lccccccc}
\hline & \multicolumn{3}{c}{ Training } & & \multicolumn{3}{c}{ Test } \\
\cline { 2 - 4 } \cline { 6 - 8 } Language & TIN & UNT & Total & & TIN & UNT & Total \\
\hline English & 149550 & 39424 & 188974 & & 850 & 1072 & 1922 \\
\hline
\end{tabular}

Table 2: Subtask B (English): statistics about the data.

\begin{tabular}{|c|c|c|c|c|c|c|c|c|}
\hline \multirow[b]{2}{*}{ Language } & \multicolumn{4}{|c|}{ Training } & \multicolumn{4}{|c|}{ Test } \\
\hline & IND & GRP & OTH & Total & IND & GRP & OTH & Total \\
\hline English & 120330 & 22176 & 7043 & 149549 & 580 & 190 & 80 & 850 \\
\hline
\end{tabular}

Table 3: Subtask C (English): statistics about the data.

\begin{tabular}{|c|c|c|c|c|}
\hline Language & Tweet & $\mathbf{A}$ & B & $\mathbf{C}$ \\
\hline English & This account owner asks for people to think rationally. & NOT & - & - \\
\hline Arabic & 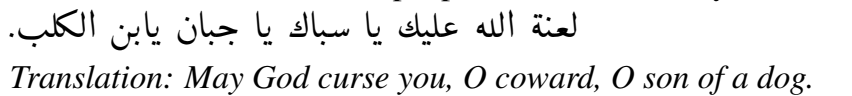 & OFF & - & - \\
\hline Danish & $\begin{array}{l}\text { Du glemmer Østeuropaer som er de værste } \\
\text { Translation: You forget Eastern Europeans, who are the worst }\end{array}$ & OFF & - & - \\
\hline Greek & 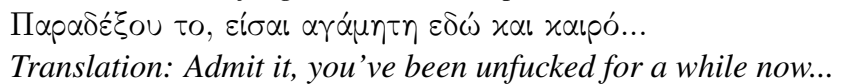 & OFF & - & - \\
\hline Turkish & $\begin{array}{l}\text { Böyle devam et seni gerizekalı } \\
\text { Translation: Go on like this, you idiot }\end{array}$ & OFF & - & - \\
\hline English & this job got me all the way fucked up real shit & OFF & UNT & - \\
\hline English & wtf ari her ass tooo big & OFF & TIN & IND \\
\hline English & @USER We are a country of morons & OFF & TIN & GRP \\
\hline
\end{tabular}

Table 4: Annotated examples for all subtasks and languages.

English For English, we provided two datasets: OLID from OffensEval-2019 (Zampieri et al., 2019a), and SOLID, which is a new dataset we created for the task (Rosenthal et al., 2020). SOLID is an abbreviation for Semi-Supervised Offensive Language Identification Dataset, and it contains 9,089,140 English tweets, which makes it the largest dataset of its kind. For SOLID, we collected random tweets using the 20 most common English stopwords such as the, of, and, to, etc. Then, we labeled the collected tweets in a semi-supervised manner using democratic co-training, with OLID as a seed dataset. For the co-training, we used four models with different inductive biases: PMI (Turney and Littman, 2003), FastText (Joulin et al., 2017), LSTM (Hochreiter and Schmidhuber, 1997), and BERT (Devlin et al., 2019). We selected the OFF tweets for the test set using this semi-supervised process and we then annotated them manually for all subtasks. We further added 2,500 NOT tweets using this process without further annotation. We computed a Fleiss' $\kappa$ Inter-Annotator Agreement (IAA) on a small subset of instances that were predicted to be OFF, and obtained 0.988 for Level A (almost perfect agreement), 0.818 for Level B (substantial agreement), and 0.630 for Level C (moderate agreement). The annotation for Level $\mathrm{C}$ was more challenging as it is 3 -way and also as sometimes there could be different types of targets mentioned in the offensive tweet, but the annotators were forced to choose only one label. 
Arabic The Arabic dataset consists of 10,000 tweets collected in April-May 2019 using the Twitter API with the language filter set to Arabic: lang: ar. In order to increase the chance of having offensive content, only tweets with two or more vocative particles ( $y A$ in Arabic) were considered for annotation; the vocative particle is used mainly to direct the speech to a person or to a group, and it is widely observed in offensive communications in almost all Arabic dialects. This yielded 20\% offensive tweets in the final dataset. The tweets were manually annotated (for Level A only) by a native speaker familiar with several Arabic dialects. A random subsample of offensive and non-offensive tweets were doubly annotated and the Fleiss $\kappa$ IAA was found to be 0.92. More details can be found in (Mubarak et al., 2020b).

Danish The Danish dataset consists of 3,600 comments drawn from Facebook, Reddit, and a local newspaper, Ekstra Bladet ${ }^{3}$. The selection of the comments was partially seeded using abusive terms gathered during a crowd-sourced lexicon compilation; in order to ensure sufficient data diversity, this seeding was limited to half the data only. The training data was not divided into distinct training/development splits, and participants were encouraged to perform cross-validation, as we wanted to avoid issues that fixed splits can cause (Gorman and Bedrick, 2019). The annotation (for Level A only) was performed at the individual comment level by males aged 25-40. A full description of the dataset and an accompanying data statement (Bender and Friedman, 2018) can be found in (Sigurbergsson and Derczynski, 2020).

Greek The Offensive Greek Twitter Dataset (OGTD) used in this task is a compilation of 10,287 tweets. These tweets were sampled using popular and trending hashtags, including television programs such as series, reality and entertainment shows, along with some politically related tweets. Another portion of the dataset was fetched using pejorative terms and "you are" as keywords. This particular strategy was adopted with the hypothesis that TV and politics would gather a handful of offensive posts, along with tweets containing vulgar language for further investigation. A team of volunteer annotators participated in the annotation process (for Level A only), with each tweet being judged by three annotators. In cases of disagreement, labels with majority agreement above $66 \%$ were selected as the actual tweet labels. The IAA was 0.78 (using Fleiss' $\kappa$ coefficient). A full description of the dataset collection and annotation is detailed in (Pitenis et al., 2020).

Turkish The Turkish dataset consists of over 35,000 tweets sampled uniformly from the Twitter stream and filtered using a list of the most frequent words in Turkish, as identified by Twitter. The tweets were annotated by volunteers (for Level A only). Most tweets were annotated by a single annotator. The Cohen's $\kappa$ IAA calculated on 5,000 doubly-annotated tweets was 0.761 . Note that we did not include any specific method for spotting offensive language, e.g., filtering by offensive words, or following usual targets of offensive language. As a result, the distribution closely resembles the actual offensive language use on Twitter, with more non-offensive tweets than offensive tweets. More details about the sampling and the annotation process can be found in (Çöltekin, 2020).

\section{Task Participation}

A total of 528 teams signed up to participate in the task, and 145 of them submitted results: 6 teams made submissions for all five languages, 19 did so for four languages, 11 worked on three languages, 13 on two languages, and 96 focused on just one language. Tables 13, 14, and 15 show a summary of which team participated in which task. A total of 70 teams submitted system description papers, which are listed in Table 12. Below, we analyze the representation and the models used for all language tracks.

Representation The vast majority of teams used some kind of pre-trained embeddings such as contextualized Transformers (Vaswani et al., 2017) and ELMo (Peters et al., 2018) embeddings. The most popular Transformers were BERT (Devlin et al., 2019), RoBERTa (Liu et al., 2019), and the multi-lingual mBERT (Devlin et al., 2019). ${ }^{4}$

\footnotetext{
${ }^{3}$ http: //ekstrabladet.dk/

${ }^{4}$ Note that there are some issues with the way mBERT processes some languages, e.g., there is no word segmentation for Arabic, the Danish åaa mapping is not handled properly (Strømberg-Derczynski et al., 2020), etc.
} 
Many teams also used context-independent embeddings from word2vec (Mikolov et al., 2013) or GloVe (Pennington et al., 2014), including language-specific embeddings such as Mazajak (Farha and Magdy, 2019) for Arabic. Some teams used other techniques: word $n$-grams, character $n$-grams, lexicons for sentiment analysis, and lexicon of offensive words. Other representations included emoji priors extracted from the weakly supervised SOLID dataset for English, and sentiment analysis using NLTK (Bird et al., 2009), Vader (Hutto and Gilbert, 2014), and FLAIR (Akbik et al., 2018).

Machine learning models In terms of machine learning models, most teams used some kind of pretrained Transformers: typically BERT, but RoBERTa, XLM-RoBERTa (Conneau et al., 2020), ALBERT (Lan et al., 2019), and GPT-2 (Radford et al., 2019) were also popular. Other popular models included CNNs (Fukushima, 1980), RNNs (Rumelhart et al., 1986), and GRUs (Cho et al., 2014). Older models such as SVMs (Cortes and Vapnik, 1995) were also used, typically as part of ensembles.

\section{English Track}

A total of 87 teams made submissions for the English track ( 23 of them participated in the 2019 edition of the task): 27 teams participated in all three English subtasks, 18 teams participated in two English subtasks, and 42 focused on one English subtask only.

Pre-processing and normalization Most teams performed some kind of pre-processing (67 teams) or text normalization (26 teams), which are typical steps when working with tweets. Text normalization included various text transformations such as converting emojis to plain text, ${ }^{5}$ segmenting hashtags, ${ }^{6}$ general tweet text normalization (Satapathy et al., 2019), abbreviation expansion, bad word replacement, error correction, lowercasing, stemming, and/or lemmatization. Other techniques included the removal of @ user mentions, URLs, hashtags, emojis, emails, dates, numbers, punctuation, consecutive character repetitions, offensive words, and/or stop words.

Additional data Most teams found the weakly supervised SOLID dataset useful, and 58 teams ended up using it in their systems. Another six teams gave it a try, but could not benefit from it, and the remaining teams only used the manually annotated training data. Some teams used additional datasets from HASOC-2019 (Mandl et al., 2019), the Kaggle competitions on Detecting Insults in Social Commentary $^{7}$ and Toxic Comment Classification ${ }^{8}$, the TRAC-2018 shared task on Aggression Identification (Kumar et al., 2018a; Kumar et al., 2018b), the Wikipedia Detox dataset (Wulczyn et al., 2017), and the datasets from (Davidson et al., 2017) and (Wulczyn et al., 2017), as well as some lexicons such as HurtLex (Bassignana et al., 2018) and Hatebase. ${ }^{9}$ Finally, one team created their own dataset.

\subsection{Subtask A}

A total of 82 teams made submissions for subtask A, and the results can be seen in Table 5. This was the most popular subtask among all subtasks and across all languages. The best team UHH-LT achieved an F1 score of 0.9204 using an ensemble of ALBERT models of different sizes. The team ranked second was UHH-LT with an F1 score of 0.9204, and it used RoBERTa-large that was fine-tuned on the SOLID dataset in an unsupervised way, i.e., using the MLM objective. The third team, Galileo, achieved an F1 score of 0.9198 , using an ensemble that combined XLM-RoBERTa-base and XLMRoBERTa-large trained on the subtask A data for all languages. The top-10 teams used BERT, RoBERTa or XLM-RoBERTa, sometimes as part of ensembles that also included CNNs and LSTMs (Hochreiter and Schmidhuber, 1997). Overall, the competition for this subtask was very strong, and the scores are very close: the teams ranked 2-16 are within one point in the third decimal place, and those ranked 2-59 are within two absolute points in the second decimal place from the best team. All but one team beat the majority class baseline (we suspect that team might have accidentally flipped their predicted labels).

\footnotetext{
${ }^{5}$ http://github.com/carpedm20/emoji

${ }^{6}$ http://github.com/grant jenks/python-wordsegment

${ }^{7}$ http://www.kaggle.com/c/detecting-insults-in-social-commentary

${ }^{8}$ http://www.kaggle.com/c/jigsaw-toxic-comment-classification-challenge

${ }^{9}$ http: //hatebase.org/
} 


\begin{tabular}{|c|c|c|c|c|c|c|c|c|}
\hline$\#$ & Team & Score & $\#$ & Team & Score & $\#$ & Team & Score \\
\hline 1 & UHH-LT & 0.9204 & 29 & UTFPR & 0.9094 & 57 & OffensSzeged & 0.9032 \\
\hline 2 & Galileo & 0.9198 & 30 & IU-UM@LING & 0.9094 & 58 & FBK-DH & 0.9032 \\
\hline 3 & Rouges & 0.9187 & 31 & TAC & 0.9093 & 59 & RGCL & 0.9006 \\
\hline 4 & GUIR & 0.9166 & 32 & SSN_NLP_MLRG & 0.9092 & 60 & byteam & 0.8994 \\
\hline 5 & KS@LTH & 0.9162 & 33 & Hitachi & 0.9091 & 61 & ANDES & 0.8990 \\
\hline 6 & kungfupanda & 0.9151 & 34 & CoLi@UdS & 0.9091 & 62 & PUM & 0.8973 \\
\hline 7 & TysonYU & 0.9146 & 35 & XD & 0.9090 & 63 & NUIG & 0.8927 \\
\hline 8 & AlexU-BackTranslation-TL & 0.9139 & 36 & UoB & 0.9090 & 64 & $\mathrm{I} 2 \mathrm{C}$ & 0.8919 \\
\hline 9 & SpurthiAH & 0.9136 & 37 & PAI-NLP & 0.9089 & 65 & sonal.kumari & 0.8900 \\
\hline 10 & amsqr & 0.9135 & 38 & PingANPAI & 0.9089 & 66 & IJS & 0.8887 \\
\hline 11 & $\mathrm{~m} 20170548$ & 0.9134 & 39 & VerifiedXiaoPAI & 0.9089 & 67 & IR3218-UI & 0.8843 \\
\hline 12 & Coffee_Latte & 0.9132 & 40 & nlpUP & 0.9089 & 68 & TeamKGP & 0.8822 \\
\hline 13 & wac81 & 0.9129 & 41 & NLP_Passau & 0.9088 & 69 & UNT Linguistics & 0.8820 \\
\hline 14 & NLPDove & 0.9129 & 42 & TheNorth & 0.9087 & 70 & janecek1 & 0.8744 \\
\hline 15 & UJNLP & 0.9128 & 43 & problemConquero & 0.9085 & 71 & Team Oulu & 0.8655 \\
\hline 16 & ARA & 0.9119 & 44 & Lee & 0.9084 & 72 & TECHSSN & 0.8655 \\
\hline 17 & Ferryman & 0.9115 & 45 & Wu427 & 0.9081 & 73 & KDELAB & 0.8653 \\
\hline 18 & ALT & 0.9114 & 46 & ITNLP & 0.9081 & 74 & HateLab & 0.8617 \\
\hline 19 & SINAI & 0.9105 & 47 & Better Place & 0.9077 & 75 & IASBS & 0.8577 \\
\hline 20 & MindCoders & 0.9105 & 48 & IIITG-ADBU & 0.9075 & 76 & IUST & 0.8288 \\
\hline 21 & IRLab_DAIICT & 0.9104 & 49 & doxaAI & 0.9075 & 77 & Duluth & 0.7714 \\
\hline 22 & erfan & 0.9103 & 50 & NTU_NLP & 0.9067 & 78 & RTNLU & 0.7665 \\
\hline 23 & Light & 0.9103 & 51 & FERMI & 0.9065 & 79 & KarthikaS & 0.6351 \\
\hline 24 & KAFK & 0.9099 & 52 & AdelaideCyC & 0.9063 & 80 & Bodensee & 0.4954 \\
\hline 25 & PALI & 0.9098 & 53 & INGEOTEC & 0.9061 & & Majority Baseline & 0.4193 \\
\hline 26 & PRHLT-UPV & 0.9097 & 54 & PGSG & 0.9060 & 81 & IRlab@IITV & 0.0728 \\
\hline 27 & YNU_oxz & 0.9097 & 55 & SRIB2020 & 0.9048 & & & \\
\hline 28 & IITP-AINLPML & 0.9094 & 56 & GruPaTo & 0.9036 & & & \\
\hline
\end{tabular}

Table 5: Results for English subtask A, ordered by macro-averaged F1 in descending order.

\subsection{Subtask B}

A total of 41 teams made submissions for subtask B, and the results can be seen in Table 6 . The best team is Galileo (which were third on subtask A), whose ensemble model achieved an F1 score of 0.7462 . The second-place team, PGSG, used a complex teacher-student architecture built on top of a BERT-LSTM model, which was fine-tuned on the SOLID dataset in an unsupervised way, i.e., optimizing for the MLM objective. NTU_NLP was ranked third with an F1 score of 0.6906. They tackled subtasks A, B, and C as part of a multi-task BERT-based model. Overall, the differences in the scores for subtask B are much larger than for subtask A. For example, the 4th team is two points behind the third one and seven points behind the first one. The top-ranking teams used BERT-based Transformer models, and all but four teams could improve over the majority class baseline.

\subsection{Subtask C}

A total of 37 teams made submissions for subtask $\mathrm{C}$ and the results are shown in Table 7. The best team was once again Galileo, with an F1 score of 0.7145. LT@Helsinki was ranked second with an F1 score of 0.6700 . They used fine-tuned BERT with oversampling to improve class imbalance. The third best system was PRHLT-UPV with an F1 score of 0.6692, which combines BERT with hand-crafted features; it is followed very closely by UHH-LT at rank 4, which achieved an F1 score of 0.6683 . This subtask is also dominated by BERT-based models, and all teams outperformed the majority class baseline.

Note that the absolute F1-scores obtained by the best teams in the English subtasks A and C are substantially higher than the scores obtained by the best teams in OffensEval-2019: 0.9223 vs. 0.8290 for subtask A and 0.7145 vs. 0.6600 for subtask C. This suggests that the much larger SOLID dataset made available in OffensEval-2020 helped the models make more accurate predictions. 


\begin{tabular}{clc|clc|llc}
\hline$\#$ & Team & Score & $\#$ & Team & Score & $\#$ & Team & Score \\
\hline 1 & Galileo & 0.7462 & 15 & Wu427 & 0.6208 & 29 & PALI & 0.5533 \\
2 & PGSG & 0.7362 & 16 & UNT Linguistics & 0.6174 & 30 & AdelaideCyC & 0.5524 \\
3 & NTU_NLP & 0.6906 & 17 & I2C & 0.6012 & 31 & KAFK & 0.5518 \\
4 & UoB & 0.6734 & 18 & PRHLT-UPV & 0.5987 & 32 & PAI-NLP & 0.5451 \\
5 & TysonYU & 0.6687 & 19 & SRIB2020 & 0.5805 & 33 & VerifiedXiaoPAI & 0.5451 \\
6 & GUIR & 0.6650 & 20 & FERMI & 0.5804 & 34 & Duluth & 0.5382 \\
7 & UHH-LT & 0.6598 & 21 & IU-UM@LING & 0.5746 & 35 & Bodensee & 0.4926 \\
8 & Ferryman & 0.6576 & 22 & PingANPAI & 0.5687 & 36 & TECHSSN & 0.3894 \\
9 & IIITG-ADBU & 0.6528 & 23 & nlpUP & 0.5687 & 37 & KarthikaS & 0.3741 \\
10 & CoLi @ UdS & 0.6445 & 24 & Team Oulu & 0.5676 & & Majority Baseline & 0.3741 \\
11 & IRLab_DAIICT & 0.6412 & 25 & KDELAB & 0.5638 & 38 & IRlab@ IITV & 0.2950 \\
12 & INGEOTEC & 0.6321 & 26 & wac81 & 0.5627 & 39 & SSN_NLP_MLRG & 0.2912 \\
13 & HateLab & 0.6303 & 27 & IITP-AINLPML & 0.5569 & 40 & IJS & 0.2841 \\
14 & AlexU-BackTranslation-TL & 0.6300 & 28 & problemConquero & 0.5569 & 41 & KEIS@ JUST & 0.2777 \\
\hline
\end{tabular}

Table 6: Results for English subtask B, ordered by macro-averaged F1 in descending order.

\begin{tabular}{clc|clc|clc}
\hline$\#$ & Team & Score & $\#$ & Team & Score & $\#$ & Team & Score \\
\hline 1 & Galileo & 0.7145 & 14 & KAFK & 0.6168 & 27 & nlpUP & 0.5515 \\
2 & LT@Helsinki & 0.6700 & 15 & ssn_nlp & 0.6116 & 28 & IS & 0.5355 \\
3 & PRHLT-UPV & 0.6692 & 16 & IJS & 0.6094 & 29 & sonal.kumari & 0.5260 \\
4 & UHH-LT & 0.6683 & 17 & PALI & 0.6015 & 30 & SRIB2020 & 0.5147 \\
5 & ITNLP & 0.6543 & 18 & FERMI & 0.5882 & 31 & KEIS@JUST & 0.4817 \\
6 & wac81 & 0.6489 & 19 & problemConquero & 0.5871 & 32 & ultraviolet & 0.4776 \\
7 & PUM & 0.6473 & 20 & Ferryman & 0.5809 & 33 & HateLab & 0.4535 \\
8 & PingANPAI & 0.6394 & 21 & AlexU-BackTranslation-TL & 0.5761 & 34 & Bodensee & 0.3462 \\
9 & IITP-AINLPML & 0.6388 & 22 & IITG-ADBU & 0.5756 & 35 & Team Oulu & 0.3220 \\
10 & PAI-NLP & 0.6347 & 23 & Duluth & 0.5744 & 36 & SSN_NLP_MLRG & 0.3178 \\
11 & GUIR & 0.6319 & 24 & KDELAB & 0.5720 & & Majority Baseline & 0.2704 \\
12 & IU-UM@LING & 0.6265 & 25 & NTU_NLP & 0.5695 & & & \\
13 & AdelaideCyC & 0.6232 & 26 & INGEOTEC & 0.5626 & & & \\
\hline
\end{tabular}

Table 7: Results for English subtask C, ordered by macro-averaged F1 in descending order.

Furthermore, it suggests that the weakly supervised method used to compile and annotate SOLID is a viable alternative to popular purely manual annotation approaches. A more detailed analysis of the systems' performances will be carried out in order to determine the contribution of the SOLID dataset for the results.

\subsection{Best Systems}

We provide some more details about the approaches used by the top teams for each subtask. We use subindices to show their rank for each subtask. Additional summaries for some of the best teams can be found in Appendix A.

Galileo (A:3, B:1, C:1) This team was ranked 3rd, 1st, and 1st on the English subtasks A, B, and C, respectively. This is also the only team ranked among the top-3 across all languages. For subtask A, they used multi-lingual pre-trained Transformers based on XLM-RoBERTa, followed by multi-lingual fine-tuning using the OffensEval data. Ultimately, they submitted an ensemble that combined XLMRoBERTa-base and XLM-RoBERTa-large, achieving an F1 score of 0.9198. For subtasks B and C, they used knowledge distillation in a teacher-student framework, using Transformers such as ALBERT and ERNIE 2.0 (Sun et al., 2020) as teacher models, achieving an F1 score of 0.7462 and 0.7145 , for subtasks $\mathrm{B}$ and $\mathrm{C}$ respectively. 


\begin{tabular}{clc|clc|clc}
\hline$\#$ & Team & Score & $\#$ & Team & Score & $\#$ & Team & Score \\
\hline 1 & ALAMIHamza & 0.9017 & 21 & SaiSakethAluru & 0.8455 & 41 & tharindu & 0.7881 \\
2 & ALT & 0.9016 & 22 & will_go & 0.8440 & 42 & PRHLT-UPV & 0.7868 \\
3 & Galileo & 0.8989 & 23 & erfan & 0.8418 & 43 & IRlab@ IITV & 0.7793 \\
4 & KUISAIL & 0.8972 & 24 & ANDES & 0.8402 & 44 & yemen2016 & 0.7721 \\
5 & AMR-KELEG & 0.8958 & 25 & Bushr & 0.8395 & 45 & saroarj & 0.7474 \\
6 & KS@LTH & 0.8902 & 26 & klaralang & 0.8241 & 46 & kxkajava & 0.7306 \\
7 & iaf7 & 0.8778 & 27 & zoher_orabe & 0.8221 & 47 & frankakorpel & 0.7251 \\
8 & INGEOTEC & 0.8744 & 28 & mircea.tanase & 0.8220 & 48 & COMA & 0.5436 \\
9 & BhamNLP & 0.8714 & 29 & machouz & 0.8216 & 49 & JCT & 0.4959 \\
10 & yasserotiefy & 0.8691 & 30 & orabia & 0.8198 & 50 & FBK-DH & 0.4642 \\
11 & SAJA & 0.8655 & 31 & Taha & 0.8183 & 51 & sonal.kumari & 0.4536 \\
12 & Ferryman & 0.8592 & 32 & hamadanayel & 0.8182 & 52 & CyberTronics & 0.4466 \\
13 & SAFA & 0.8555 & 33 & CoLi @ UdS & 0.8176 & 53 & SpurthiAH & 0.4451 \\
14 & hhaddad & 0.8520 & 34 & fatemah & 0.8147 & & Majority Baseline & 0.4441 \\
15 & TAC & 0.8519 & 35 & jbern & 0.8125 & & & \\
16 & saradhix & 0.8500 & 36 & zahra.raj & 0.8057 & & & \\
17 & lukez & 0.8498 & 37 & I2C & 0.8056 & & & \\
18 & Rouges & 0.8480 & 38 & jlee24282 & 0.8024 & & & \\
19 & TysonYU & 0.8474 & 39 & problemConquero & 0.8021 & & & \\
20 & NLPDove & 0.8455 & 40 & asking28 & 0.8002 & & & \\
\hline
\end{tabular}

Table 8: Results for Arabic subtask A, ordered by macro-averaged F1 in descending order.

UHH-LT (A:1) This team was ranked 1st on subtask A with an F1 score of 0.9223. They fine-tuned different Transformer models on the OLID training data, and then combined them into an ensemble. They experimented with BERT-base and BERT-large (uncased), RoBERTa-base and RoBERTa-large, XLMRoBERTa, and four different ALBERT models (large-v1, large-v2, xxlarge-v1, and xxlarge-v2). In their official submission, they used an ensemble combining different ALBERT models. They did not use the labels of the SOLID dataset, but found the tweets it contained nevertheless useful for unsupervised fine-tuning (i.e., using the MLM objective) of the pre-trained Transformers.

\section{Arabic Track}

A total of 108 teams registered to participate in the Arabic track, and ultimately 53 teams entered the competition with at least one valid submission. Among them, ten teams participated in the Arabic track only, while the rest participated in other languages in addition to Arabic. This was the second shared task for Arabic after the one at the 4th workshop on Open-Source Arabic Corpora and Processing Tools (Mubarak et al., 2020a), which had different settings and less participating teams.

Pre-processing and normalization Most teams performed some kind of pre-processing or text normalization, e.g., Hamza shapes, Alif Maqsoura, Taa Marbouta, diacritics, non-Arabic characters, etc., and only one team replaced emojis with their textual counter-parts.

\subsection{Results}

Table 8 shows the teams and the F1 scores they achieved for the Arabic subtask A. The majority class baseline had an F1 score of 0.4441 , and several teams achieved results that doubled that baseline score. The best-performing team was ALAMIHamza with an F1 score of 0.9017. The second-best team, ALT, was almost tied with the winner, with an F1 score of 0.9016. The Galileo team was third with an F1 score of 0.8989. A summary of the approaches taken by the top-performing teams can be found in Appendix A; here we briefly describe the winning system:

ALAMIHamza (A: 1) The winning team achieved the highest F1-score using BERT to encode Arabic tweets, followed by a sigmoid classifier. They further performed translation of the meaning of emojis. 


\begin{tabular}{clc|clc|llc}
\hline$\#$ & Team & Score & $\#$ & Team & Score & $\#$ & Team & Score \\
\hline 1 & LT@Helsinki & 0.8119 & 14 & Rouges & 0.7587 & 27 & TeamKGP & 0.6973 \\
2 & Galileo & 0.8021 & 14 & Smatgrisene & 0.7587 & 28 & Stormbreaker & 0.6842 \\
3 & NLPDove & 0.7923 & 16 & machouz & 0.7561 & 29 & TAC & 0.6819 \\
4 & FBK-DH & 0.7766 & 17 & IU-UM@LING & 0.7553 & 30 & Sonal & 0.6711 \\
5 & KS@LTH & 0.7750 & 18 & Ferryman & 0.7525 & 31 & RGCL & 0.6556 \\
6 & JCT & 0.7741 & 19 & MindCoders & 0.7380 & 32 & PRHLT-UPV & 0.6369 \\
7 & ANDES & 0.7723 & 20 & ARA & 0.7267 & 33 & IUST & 0.6226 \\
8 & TysonYU & 0.7685 & 21 & INGEOTEC & 0.7237 & 34 & SRIB2020 & 0.6127 \\
8 & FERMI & 0.7685 & 22 & KUISAIL & 0.7231 & 35 & IR3218-UI & 0.5736 \\
10 & NLP_Passau & 0.7673 & 23 & JAK & 0.7086 & 36 & SSN_NLP_MLRG & 0.5678 \\
11 & GruPaTo & 0.7620 & 24 & LIIR & 0.7019 & 37 & Team Oulu & 0.5587 \\
12 & KEIS@JUST & 0.7612 & 25 & MeisterMorxrc & 0.6998 & 38 & IJS & 0.4913 \\
13 & will_go & 0.7596 & 26 & problemConquero & 0.6974 & & Majority Baseline & 0.4668 \\
\hline
\end{tabular}

Table 9: Results for Danish subtask A, ordered by macro-averaged F1 in descending order.

\section{Danish Track}

A total of 72 teams registered to participate in the Danish track, and 39 of them actually made official submissions on the test dataset. This is the first shared task on offensive language identification to include Danish, and the dataset provided to the OffensEval-2020 participants is an extended version of the one from (Sigurbergsson and Derczynski, 2020).

Pre-processing and normalization Many teams used the pre-processing included in the relevant embedding model, e.g., BPE (Heinzerling and Strube, 2018) and WordPiece. Other pre-processing techniques included emoji normalization, spelling correction, sentiment tagging, lexical and regex-based term and phrase flagging, and hashtag segmentation.

\subsection{Results}

The results are shown in Table 9. We can see that all teams managed to outperform the majority class baseline. Moreover, all but one team improved over a FastText baseline $(\mathrm{F} 1=0.5148)$, and most teams achieved an F1 score of 0.7 or higher. Interestingly, one of the top-ranked teams, JCT, was entirely non-neural.

LT @Helsinki (A: 1) The winning team LT@ Helsinki used NordicBERT for representation, as provided by BotXO. ${ }^{10}$ NordicBERT is customized to Danish, and avoids some of the pre-processing noise and ambiguity introduced by other popular BERT implementations. The team further reduced orthographic lengthening to maximum two repeated characters, converted emojis to sentiment scores, and used cooccurrences of hashtags and references to usernames. They tuned the hyper-parameters of their model using 10-fold cross validation.

\section{Greek Track}

A total of 71 teams registered to participate in the Greek track, and ultimately 37 of them made an official submission on the test dataset. This is the first shared task on offensive language identification to include Greek, and the dataset provided to the OffensEval-2020 participants is an extended version of the one from (Pitenis et al., 2020).

Pre-processing and normalization The participants experimented with various pre-processing and text normalization techniques, similarly to what was done for the other languages above. One team further reported replacement of emojis with their textual equivalent.

\footnotetext{
${ }^{10}$ See http://github.com/botxo/nordic_bert
} 


\begin{tabular}{clc|clc|clc}
\hline$\#$ & Team & Score & $\#$ & Team & Score & $\#$ & Team & Score \\
\hline 1 & NLPDove & 0.8522 & 14 & CoLi @ UdS & 0.8147 & 27 & IUST & 0.7756 \\
2 & Galileo & 0.8507 & 15 & TAC & 0.8141 & 28 & KEIS@JUST & 0.7730 \\
3 & KS@LTH & 0.8481 & 16 & IU-UM@LING & 0.8140 & 29 & FBK-DH & 0.7700 \\
4 & KUISAIL & 0.8432 & 17 & MindCoders & 0.8137 & 30 & Team Oulu & 0.7615 \\
5 & IJS & 0.8329 & 18 & RGCL & 0.8135 & 31 & JCT & 0.7568 \\
6 & SU-NLP & 0.8317 & 19 & problemConquero & 0.8115 & 32 & IRlab@IITV & 0.7181 \\
7 & LT@Helsinki & 0.8258 & 20 & Rouges & 0.8030 & 33 & TeamKGP & 0.7041 \\
8 & FERMI & 0.8231 & 21 & TysonYU & 0.8022 & 34 & SSN_NLP_MLRG & 0.6779 \\
9 & Ferryman & 0.8222 & 22 & Sonal & 0.8017 & 35 & fatemah & 0.6036 \\
10 & INGEOTEC & 0.8197 & 23 & JAK & 0.7956 & 36 & CyberTronics & 0.4265 \\
11 & will_go & 0.8176 & 24 & ARA & 0.7828 & & Majority Baseline & 0.4202 \\
12 & ANDES & 0.8153 & 25 & machouz & 0.7820 & 37 & Stormbreaker & 0.2688 \\
13 & LIIR & 0.8148 & 26 & PRHLT-UPV & 0.7763 & & & \\
\hline
\end{tabular}

Table 10: Results for Greek subtask A, ordered by macro-averaged F1 in descending order.

\subsection{Results}

The evaluation results are shown in Table 10. The top team, NLPDove, achieved an F1 score of 0.852 , with Galileo coming close at the second place with an F1 score of 0.851. The KS@LTH team was ranked third with an F1 score of 0.848 . It is no surprise that the majority of the high-ranking submissions and participants used large-scale pre-trained Transformers, with BERT being the most prominent among them, along with wordwvec-style non-contextualized pre-trained word embeddings.

NLPDove (A:1) The winning team NLPDove used pre-trained word embeddings from mBERT, which they fine-tuned using the training data. A domain-specific vocabulary was generated by running the WordPiece algorithm (Schuster and Nakajima, 2012) and using embeddings for extended vocabulary to pre-train and fine-tune the model.

\section{Turkish Track}

A total of 86 teams registered to participate in the Turkish track, and ultimately 46 of them made an official submission on the test dataset. All teams except for one participated in at least one other track. This is the first shared task on offensive language identification to include Turkish, and the dataset provided to the OffensEval-2020 participants is an extended version of the one from (Çöltekin, 2020).

\subsection{Results}

The results are shown in Table 11. We can see that team Galileo achieved the highest macro-averaged F1 score of 0.8258 , followed by SU-NLP and KUI-SAIL with F1 scores of 0.8167 and 0.8141 , respectively. Note that the latter two teams are from Turkey, and they used some language-specific resources and tuning. Most results were in the interval $0.7-0.8$, and almost all teams managed to outperform the majority class baseline, which had an F1 score of 0.4435 .

Galileo (A:1) The best team in the Turkish subtask A was Galileo, which achieved top results in several other tracks. Unlike the systems ranked second and third, Galileo's system is language-agnostic, and it used data for all five languages in a multi-lingual training setup.

\section{Conclusion and Future Work}

We presented the results of OffensEval-2020, which featured datasets in five languages: Arabic, Danish, English, Greek, and Turkish. For English, we had three subtasks, representing the three levels of the OLID hierarchy. For the other four languages, we had a subtask for the top-level of the OLID hierarchy only. A total of 528 teams signed up to participate in OffensEval-2020, and 145 of them actually submitted results across all languages and subtasks. 


\begin{tabular}{rlr|rlr|rlr}
\hline$\#$ & Team & Score & $\#$ Team & Score & $\#$ & Team & Score \\
\hline 1 & Galileo & 0.8258 & 18 & LT@Helsinki & 0.7719 & 35 & PRHLT-UPV & 0.7127 \\
2 & SU-NLP & 0.8167 & 19 & NLP_Passau & 0.7676 & 36 & SRIB2020 & 0.6993 \\
3 & KUISAIL & 0.8141 & 20 & will_go & 0.7653 & 37 & Team Oulu & 0.6868 \\
4 & KS@LTH & 0.8101 & 21 & FERMI & 0.7578 & 38 & ARA & 0.6381 \\
5 & NLPDove & 0.7967 & 22 & problemConquero & 0.7553 & 39 & DH-FBK & 0.6268 \\
6 & TysonYU & 0.7933 & 23 & pin_cod_ & 0.7496 & 40 & f_shahaby & 0.5730 \\
7 & RGCL & 0.7859 & 24 & TAC & 0.7477 & 41 & CyberTronics & 0.5420 \\
8 & Rouges & 0.7815 & 25 & IUST & 0.7476 & 42 & IASBS & 0.5362 \\
9 & GruPaTo & 0.7790 & 26 & alaeddin & 0.7473 & 43 & JCT & 0.5099 \\
10 & MindCoders & 0.7789 & 27 & fatemah & 0.7469 & 44 & machouz & 0.4518 \\
11 & INGEOTEC & 0.7758 & 28 & CoLi @ UdS & 0.7461 & 45 & jooyeon Lee & 0.4435 \\
12 & Ferryman & 0.7737 & 29 & Sonal & 0.7422 & & Majority Baseline & 0.4435 \\
13 & ANDES & 0.7737 & 30 & MeisterMorxrc & 0.7398 & 46 & Stormbreaker & 0.3109 \\
14 & I2C & 0.7735 & 31 & JAK & 0.7334 & & & \\
15 & IU-UM@LING & 0.7729 & 32 & KEIS@JUST & 0.7330 & & & \\
16 & IJS & 0.7724 & 33 & TeamKGP & 0.7301 & & & \\
17 & LIIR & 0.7720 & 34 & TOBB ETU & 0.7154 & & & \\
\hline
\end{tabular}

Table 11: Results for Turkish subtask A, ordered by macro-averaged F1 in descending order.

Out of the 145 participating teams, 96 teams participated in one language only, 13 teams participated in two languages, 11 in three languages, 19 in four languages, and 6 teams submitted systems for all five languages. The official submissions per language ranged from 37 (for Greek) to 81 (for English). Finally, 70 of the 145 participating teams submitted system description papers, which is an all-time record.

The wide participation in the task allowed us to compare a number of approaches across different languages and datasets. Similarly to OffensEval-2019, we observed that the best systems for all languages and subtasks used large-scale BERT-style pre-trained Transformers such as BERT, RoBERTa, and mBERT. Unlike 2019, however, the multi-lingual nature of this year's data enabled cross-language approaches, which proved quite effective and were used by some of the top-ranked systems.

In future work, we plan to extend the task in several ways. First, we want to offer subtasks B and C for all five languages from OffensEval-2020. We further plan to add some additional languages, especially under-represented ones. Other interesting aspects to explore are code-mixing, e.g., mixing Arabic script and Latin alphabet in the same Arabic message, and code-switching, e.g., mixing Arabic and English words and phrases in the same message. Last but not least, we plan to cover a wider variety of social media platforms.

\section{Acknowledgements}

This research was partly supported by the IT University of Copenhagen's Abusive Language Detection project. It is also supported by the Tanbih project at the Qatar Computing Research Institute, HBKU, which aims to limit the effect of "fake news," propaganda and media bias by making users aware of what they are reading.

\section{References}

Hwijeen Ahn, Jimin Sun, Chan Young Park, and Jungyun Seo. 2020. NLPDove at SemEval-2020 Task 12: Improving offensive language detection with cross-lingual transfer. In Proceedings of the International Workshop on Semantic Evaluation (SemEval).

Alan Akbik, Duncan Blythe, and Roland Vollgraf. 2018. Contextual string embeddings for sequence labeling. In Proceedings of the International Conference on Computational Linguistics (COLING).

Hamza Alami, Said Ouatik El Alaoui, Abdessamad Benlahbib, and Noureddine En-nahnahi. 2020. LISAC FSDMUSMBA Team at SemEval 2020 Task 12: Overcoming AraBERT's pretrain-finetune discrepancy for Arabic offensive language identification. In Proceedings of the International Workshop on Semantic Evaluation (SemEval). 
Abdullah I. Alharbi and Mark Lee. 2020. BhamNLP at SemEval-2020 Task 12: An ensemble of different word embeddings and emotion transfer learning for Arabic offensive language identification in social media. In Proceedings of the International Workshop on Semantic Evaluation (SemEval).

Pedro Alonso, Rajkumar Saini, and György Kovacs. 2020. TheNorth at SemEval-2020 Task 12: Hate speech detection using RoBERTa. In Proceedings of the International Workshop on Semantic Evaluation (SemEval).

Talha Anwar and Omer Baig. 2020. TAC at SemEval-2020 Task 12: Ensembling approach for multilingual offensive language identification in social media. In Proceedings of the International Workshop on Semantic Evaluation (SemEval).

Aymé Arango, Juan Manuel Pérez, and Franco Luque. 2020. ANDES at SemEval-2020 Task 12: A single BERT multilingual model for offensive language detection. In Proceedings of the International Workshop on Semantic Evaluation (SemEval).

Pinar Arslan. 2020. pin_cod_ at SemEval-2020 Task 12: Injecting lexicons into bidirectional long short-term memory networks to detect Turkish offensive tweets. In Proceedings of the International Workshop on Semantic Evaluation (SemEval).

Arup Baruah, Kaushik Das, Ferdous Barbhuiya, and Kuntal Dey. 2020. IIITG-ADBU at SemEval-2020 Task 12: Comparison of BERT and BiLSTM in detecting offensive language. In Proceedings of the International Workshop on Semantic Evaluation (SemEval).

Valerio Basile, Cristina Bosco, Elisabetta Fersini, Debora Nozza, Viviana Patti, Francisco Manuel Rangel Pardo, Paolo Rosso, and Manuela Sanguinetti. 2019. SemEval-2019 task 5: Multilingual detection of hate speech against immigrants and women in Twitter. In Proceedings of the International Workshop on Semantic Evaluation (SemEval).

Elisa Bassignana, Valerio Basile, and Viviana Patti. 2018. Hurtlex: A multilingual lexicon of words to hurt. In Proceedings of the Fifth Italian Conference on Computational Linguistics (CLiC-it).

Emily M Bender and Batya Friedman. 2018. Data statements for natural language processing: Toward mitigating system bias and enabling better science. Transactions of the Association for Computational Linguistics, 6:587604.

Steven Bird, Ewan Klein, and Edward Loper. 2009. Natural language processing with Python: analyzing text with the natural language toolkit. O'Reilly.

Marcos Boriola and Gustavo Paetzold. 2020. UTFPR at SemEval-2020 Task 12: Identifying offensive tweets with lightweight ensembles. In Proceedings of the International Workshop on Semantic Evaluation (SemEval).

Camilla Casula, Stefano Menini, Alessio Palmero Aprosio, and Sara Tonelli. 2020. DH-FBK at SemEval-2020 Task 12: Using multi-channel BERT for multilingual offensive language detection. In Proceedings of the International Workshop on Semantic Evaluation (SemEval).

Çağrı Çöltekin. 2020. A corpus of Turkish offensive language on social media. In Proceedings of the 12th International Conference on Language Resources and Evaluation (LREC).

Kathryn Chapman, Johannes Bernhard, and Dietrich Klakow. 2020. CoLi @ UdS at SemEval-2020 Task 12: Offensive tweet detection with ensembling. In Proceedings of the International Workshop on Semantic Evaluation (SemEval).

Po-Chun Chen, Hen-Hsen Huang, and Hsin-Hsi Chen. 2020. NTU_NLP at SemEval-2020 Task 12: Identifying offensive tweets using hierarchical multi-task learning approach. In Proceedings of the International Workshop on Semantic Evaluation (SemEval).

Kyunghyun Cho, Bart van Merriënboer, Caglar Gulcehre, Dzmitry Bahdanau, Fethi Bougares, Holger Schwenk, and Yoshua Bengio. 2014. Learning phrase representations using RNN encoder-decoder for statistical machine translation. In Proceedings of the Conference on Empirical Methods in Natural Language Processing (EMNLP).

Davide Colla, Tommaso Caselli, Valerio Basile, Jelena Mitrović, and Michael Granitzer. 2020. GruPaTo at SemEval-2020 Task 12: Retraining mBERT on social media and fine-tuned offensive language models. In Proceedings of the International Workshop on Semantic Evaluation (SemEval).

Alexis Conneau, Kartikay Khandelwal, Naman Goyal, Vishrav Chaudhary, Guillaume Wenzek, Francisco Guzmán, Edouard Grave, Myle Ott, Luke Zettlemoyer, and Veselin Stoyanov. 2020. Unsupervised crosslingual representation learning at scale. In Proceedings of the 58th Annual Meeting of the Association for Computational Linguistics (ACL).

Corinna Cortes and Vladimir Vapnik. 1995. Support-vector networks. Machine learning, 20(3):273-297.

Tanvi Dadu and Kartikey Pant. 2020. Team Rouges at SemEval-2020 Task 12: Cross-lingual inductive transfer to detect offensive language. In Proceedings of the International Workshop on Semantic Evaluation (SemEval).

Wenliang Dai, Tiezheng Yu, Zihan Liu, and Pascale Fung. 2020. Kungfupanda at SemEval-2020 Task 12: BERTbased multi-task learning for offensive language detection. In Proceedings of the International Workshop on Semantic Evaluation (SemEval). 
Kaushik Amar Das, Arup Baruah, Ferdous Ahmed Barbhuiya, and Kuntal Dey. 2020. KAFK at SemEval-2020 Task 12: Checkpoint ensemble of transformers for hate speech classification. In Proceedings of the International Workshop on Semantic Evaluation (SemEval).

Thomas Davidson, Dana Warmsley, Michael Macy, and Ingmar Weber. 2017. Automated hate speech detection and the problem of offensive language. In Proceedings of the 11th International AAAI Conference on Web and Social Media, ICWSM '17.

Gretel Liz De la Peña Sarracén and Paolo Rosso. 2020. PRHLT-UPV at SemEval-2020 Task 12: BERT for multilingual offensive language detection. In Proceedings of the International Workshop on Semantic Evaluation (SemEval).

Jacob Devlin, Ming-Wei Chang, Kenton Lee, and Kristina Toutanova. 2019. BERT: Pre-training of deep bidirectional transformers for language understanding. In Proceedings of the Conference of the North American Chapter of the Association for Computational Linguistics: Human Language Technologies (NAACL-HLT).

Xiangjue Dong and Jinho D. Choi. 2020. XD at SemEval-2020 Task 12: Ensemble approach to offensive language identification in social media using transformer encoders. In Proceedings of the International Workshop on Semantic Evaluation (SemEval).

Ibrahim Abu Farha and Walid Magdy. 2019. Mazajak: An online Arabic sentiment analyser. In Proceedings of the Fourth Arabic Natural Language Processing Workshop (WANLP).

Jared Fromknecht and Alexis Palmer. 2020. UNT Linguistics at OffensEval 2020: Linear SVC with pre-trained word embeddings as document vectors and targeted linguistic features. In Proceedings of the International Workshop on Semantic Evaluation (SemEval).

Kunihiko Fukushima. 1980. Neocognitron: A self-organizing neural network model for a mechanism of pattern recognition unaffected by shift in position. Biological cybernetics, 36(4):193-202.

Avishek Garain. 2020. Garain at SemEval-2020 Task 12: Sequence based deep learning for categorizing offensive language in social media. In Proceedings of the International Workshop on Semantic Evaluation (SemEval).

Erfan Ghadery and Marie-Francine Moens. 2020. LIIR at SemEval-2020 Task 12: A cross-lingual augmentation approach for multilingual offensive language identification. In Proceedings of the International Workshop on Semantic Evaluation (SemEval).

Soumitra Ghosh, Asif Ekbal, and Pushpak Bhattacharyya. 2020. IITP-AINLPML at SemEval-2020 Task 12: Offensive tweet identification and target categorization in a multitask environment. In Proceedings of the International Workshop on Semantic Evaluation (SemEval).

Kyle Gorman and Steven Bedrick. 2019. We need to talk about standard splits. In Proceedings of the 57th Annual Meeting of the Association for Computational Linguistics (ACL).

Ehab Hamdy, Jelena Mitrović, and Michael Granitzer. 2020. nlpUP at SemEval-2020 Task 12: A blazing fast system for offensive language detection. In Proceedings of the International Workshop on Semantic Evaluation (SemEval).

Keisuke Hanahata and Masaki Aono. 2020. KDELAB at SemEval-2020 Task 12: A system for estimating aggression of tweets using two layers of BERT features. In Proceedings of the International Workshop on Semantic Evaluation (SemEval).

Sabit Hassan, Younes Samih, Hamdy Mubarak, and Ahmed Abdelali. 2020. ALT at SemEval-2020 Task 12: Arabic and English offensive language identification in social media. In Proceedings of the International Workshop on Semantic Evaluation (SemEval).

Benjamin Heinzerling and Michael Strube. 2018. BPEmb: Tokenization-free pre-trained subword embeddings in 275 languages. In Proceedings of the 11th International Conference on Language Resources and Evaluation.

Peter Juel Henrichsen and Marianne Rathje. 2020. Smatgrisene at SemEval-2020 Task 12: Offense detection by AI - with a pinch of real I. In Proceedings of the International Workshop on Semantic Evaluation (SemEval).

Mahen Herath, Thushari Atapattu, Dung Anh Hoang, Christoph Treude, and Katrina Falkner. 2020. AdelaideCyC at SemEval-2020 Task 12: Ensemble of classifiers for offensive language detection in social media. In Proceedings of the International Workshop on Semantic Evaluation (SemEval).

Sepp Hochreiter and Jürgen Schmidhuber. 1997. Long short-term memory. Neural computation, 9(8):1735-1780.

Fatemah Husain, Jooyeon Lee, Samuel Henry, and Ozlem Uzuner. 2020. SalamNET at SemEval-2020 Task 12: Deep learning approach for Arabic offensive language detection. In Proceedings of the International Workshop on Semantic Evaluation (SemEval).

Omar Hussein, Hachem Sfar, Jelena Mitrović, and Michael Granitzer. 2020. NLP_Passau at SemEval-2020 Task 12: Multilingual neural network for offensive language detection in English, Danish and Turkish. In Proceedings of the International Workshop on Semantic Evaluation (SemEval). 
Clayton J Hutto and Eric Gilbert. 2014. VADER: A parsimonious rule-based model for sentiment analysis of social media text. In Proceedings of the 8th International AAAI Conference on Weblogs and Social Media (ICWSM).

Mai Ibrahim, Marwan Torki, and Nagwa El-Makky. 2020. AlexU-BackTranslation-TL at SemEval-2020 Task 12: Improving offensive language detection using data augmentation and transfer learning. In Proceedings of the International Workshop on Semantic Evaluation (SemEval).

Md Saroar Jahan and Mourad Oussalah. 2020. Team Oulu at SemEval-2020 Task 12: Multilingual identification of offensive language, type and target of Twitter post using translated datasets. In Proceedings of the International Workshop on Semantic Evaluation (SemEval).

Piotr Janiszewski, Mateusz Skiba, and Urszula Walińska. 2020. PUM at SemEval-2020 Task 12: Aggregation of Transformer-based models' features for offensive language recognition. In Proceedings of the International Workshop on Semantic Evaluation (SemEval).

Armand Joulin, Edouard Grave, Piotr Bojanowski, and Tomas Mikolov. 2017. Bag of tricks for efficient text classification. In Proceedings of the 15th Conference of the European Chapter of the Association for Computational Linguistics (EACL).

Li Junyi, Zhou Xiaobing, and Zhang Zichen. 2020. Lee at SemEval-2020 Task 12: A BERT model based on the maximum self-ensemble strategy for identifying offensive. In Proceedings of the International Workshop on Semantic Evaluation (SemEval).

A. Kalaivani and Thenmozhi D. 2020. SSN_NLP_MLRG at SemEval-2020 Task 12: Offensive language identification in English, Danish, Greek using BERT and machine learning approach. In Proceedings of the International Workshop on Semantic Evaluation (SemEval).

Ritesh Kumar, Atul Kr. Ojha, Shervin Malmasi, and Marcos Zampieri. 2018a. Benchmarking aggression identification in social media. In Proceedings of the First Workshop on Trolling, Aggression and Cyberbulling (TRAC).

Ritesh Kumar, Aishwarya N. Reganti, Akshit Bhatia, and Tushar Maheshwari. 2018b. Aggression-annotated Corpus of Hindi-English Code-mixed Data. In Proceedings of the Eleventh International Conference on Language Resources and Evaluation (LREC 2018).

Ritesh Kumar, Atul Kr. Ojha, Shervin Malmasi, and Marcos Zampieri. 2020. Evaluating Aggression Identification in Social Media. In Proceedings of the Second Workshop on Trolling, Aggression and Cyberbulling (TRAC), Santa Fe, USA.

Sonal Kumari. 2020. Sonal.kumari at SemEval-2020 Task 12: Social media multilingual offensive text identification and categorization using neural network models. In Proceedings of the International Workshop on Semantic Evaluation (SemEval).

Sandy Kurniawan, Indra Budi, and Muhammad Okky Ibrohim. 2020. IR3218-UI at SemEval-2020 Task 12: Emoji effects on offensive language identification. In Proceedings of the International Workshop on Semantic Evaluation (SemEval).

Zhenzhong Lan, Mingda Chen, Sebastian Goodman, Kevin Gimpel, Piyush Sharma, and Radu Soricut. 2019. ALBERT: A lite BERT for self-supervised learning of language representations. arXiv preprint arXiv:1909.11942.

Karishma Laud, Jagriti Singh, Randeep Kumar Sahu, and Ashutosh Modi. 2020. problemConquero at SemEval2020 Task 12: Transformer and soft label-based approaches. In Proceedings of the International Workshop on Semantic Evaluation (SemEval).

Wah Meng Lim and Harish Tayyar Madabushi. 2020. UoB at SemEval-2020 Task 6: Boosting BERT with corpus level information. In Proceedings of the International Workshop on Semantic Evaluation (SemEval).

Yinhan Liu, Myle Ott, Naman Goyal, Jingfei Du, Mandar Joshi, Danqi Chen, Omer Levy, Mike Lewis, Luke Zettlemoyer, and Veselin Stoyanov. 2019. RoBERTa: A robustly optimized BERT pretraining approach. $a r X i v$ preprint arXiv:1907.11692.

Thomas Mandl, Sandip Modha, Prasenjit Majumder, Daksh Patel, Mohana Dave, Chintak Mandlia, and Aditya Patel. 2019. Overview of the HASOC Track at FIRE 2019: Hate speech and offensive content identification in Indo-European languages. In Proceedings of the 11th Forum for Information Retrieval Evaluation (FIRE).

Abir Messaoudi, Hatem Haddad, and Moez Ben Haj Hmida. 2020. iCompass at SemEval-2020 Task 12: From a syntax-ignorant n-gram embeddings model to a deep bidirectional language model. In Proceedings of the International Workshop on Semantic Evaluation (SemEval).

Tomas Mikolov, Ilya Sutskever, Kai Chen, Greg S Corrado, and Jeff Dean. 2013. Distributed representations of words and phrases and their compositionality. In Proceedings of Advances in Neural Information Processing Systems (NIPS).

Sabino Miranda-Jiménez, Eric S. Tellez, Mario Graff, and Daniela Moctezuma. 2020. INGEOTEC at SemEval2020 Task 12: Multilingual classification of offensive text. In Proceedings of the International Workshop on Semantic Evaluation (SemEval). 
Alejandro Mosquera. 2020. amsqr at SemEval-2020 Task 12: Offensive language detection using neural networks and anti-adversarial features. In Proceedings of the International Workshop on Semantic Evaluation (SemEval).

Hamdy Mubarak, Kareem Darwish, Walid Magdy, Tamer Elsayed, and Hend Al-Khalifa. 2020a. Overview of OSACT4 Arabic offensive language detection shared task. In Proceedings of the 4th Workshop on Open-Source Arabic Corpora and Processing Tools, with a Shared Task on Offensive Language Detection.

Hamdy Mubarak, Ammar Rashed, Kareem Darwish, Younes Samih, and Ahmed Abdelali. 2020b. Arabic offensive language on Twitter: Analysis and experiments. arXiv preprint arXiv:2004.02192.

Hamada A. Nayel. 2020. NAYEL at SemEval-2020 Task 12: TF/IDF-based approach for automatic offensive language detection in Arabic tweets. In Proceedings of the International Workshop on Semantic Evaluation (SemEval).

Zoher Orabe, Bushr Haddad, Nada Ghneim, and Anas Al-Abood. 2020. DoTheMath at SemEval-2020 Task 12: Deep neural networks with self attention for Arabic offensive language detection. In Proceedings of the International Workshop on Semantic Evaluation (SemEval).

Yasser Otiefy, Ahmed Abdelmalek, and Islam El Hosary. 2020. WOLI at SemEval-2020 Task 12: Arabic offensive language identification on different Twitter datasets. In Proceedings of the International Workshop on Semantic Evaluation (SemEval).

Xiaozhi Ou, Xiaobing Zhou, and Xuejie Zhang. 2020. YNU_oxz at SemEval-2020 Task 12: Bidirectional GRU with capsule for identifying multilingual offensive language. In Proceedings of the International Workshop on Semantic Evaluation (SemEval).

Marc Pàmies, Emily Öhman, Kaisla Kajava, and Jörg Tiedemann. 2020. LT@Helsinki at SemEval-2020 Task 12: Multilingual or language-specific BERT? In Proceedings of the International Workshop on Semantic Evaluation (SemEval).

Apurva Parikh, Abhimanyu Singh Bisht, and Prasenjit Majumder. 2020. IRLab_DAIICT at SemEval-2020 Task 12: Machine learning and deep learning methods for offensive language identification. In Proceedings of the International Workshop on Semantic Evaluation (SemEval).

Ted Pedersen. 2020. Duluth at SemEval-2020 Task 12: Offensive tweet identification in English with logistic regression. In Proceedings of the International Workshop on Semantic Evaluation (SemEval).

Jeffrey Pennington, Richard Socher, and Christopher D. Manning. 2014. GloVe: Global vectors for word representation. In Proceedings of the Conference on Empirical Methods in Natural Language Processing (EMNLP).

Matthew E Peters, Mark Neumann, Mohit Iyyer, Matt Gardner, Christopher Clark, Kenton Lee, and Luke Zettlemoyer. 2018. Deep contextualized word representations. In Proceedings of the Conference of the North American Chapter of the Association for Computational Linguistics: Human Language Technology (NAACL-HLT).

Bao-Tran Pham-Hong and Setu Chokshi. 2020. PGSG at SemEval-2020 Task 12: BERT-LSTM with tweets' pretrained model and noisy student training method. In Proceedings of the International Workshop on Semantic Evaluation (SemEval).

Zeses Pitenis, Marcos Zampieri, and Tharindu Ranasinghe. 2020. Offensive language identification in Greek. In Proceedings of the 12th Language Resources and Evaluation Conference (LREC).

Flor Miriam Plaza-del Arco, M. Dolores Molina-González, L. Alfonso Ureña-López, and M. Teresa MartínValdivia. 2020. SINAI at SemEval-2020 Task 12: Offensive language identification exploring transfer learning models. In Proceedings of the International Workshop on Semantic Evaluation (SemEval).

Alec Radford, Jeffrey Wu, Rewon Child, David Luan, Dario Amodei, and Ilya Sutskever. 2019. Language models are unsupervised multitask learners. OpenAI blog, 1(8).

Tharindu Ranasinghe and Hansi Hettiarachchi. 2020. BRUMS at SemEval-2020 Task 12: Transformer based multilingual offensive language identification in social media. In Proceedings of the International Workshop on Semantic Evaluation (SemEval).

Manikandan Ravikiran, Amin Ekant Muljibhai, Toshinori Miyoshi, Hiroaki Ozaki, Yuta Koreeda, and Sakata Masayuki. 2020. Hitachi at SemEval-2020 Task 12: Offensive language identification with noisy labels using statistical sampling and post-processing. In Proceedings of the International Workshop on Semantic Evaluation (SemEval).

Sara Rosenthal, Pepa Atanasova, Georgi Karadzhov, Marcos Zampieri, and Preslav Nakov. 2020. A large-scale semi-supervised dataset for offensive language identification. arXiv preprint arXiv:2004.14454.

David E Rumelhart, Geoffrey E Hinton, and Ronald J Williams. 1986. Learning representations by backpropagating errors. Nature, 323(6088):533-536.

Ali Safaya, Moutasem Abdullatif, and Deniz Yuret. 2020. KUISAIL at SemEval-2020 Task 12: BERT-CNN for offensive speech identification in social media. In Proceedings of the International Workshop on Semantic Evaluation (SemEval). 
Anita Saroj, Supriya Chanda, and Sukomal Pal. 2020. IRlab@IITV at SemEval-2020 Task 12: multilingual offensive language identification in social media using SVM. In Proceedings of the International Workshop on Semantic Evaluation (SemEval).

Ranjan Satapathy, Yang Li, Sandro Cavallari, and Erik Cambria. 2019. Seq2seq deep learning models for microtext normalization. In Proceedings of the International Joint Conference on Neural Networks (IJCNN).

Paul Sayanta, Saha Sriparna, and Hasanuzzaman Mohammed. 2020. CyberTronics at SemEval-2020 Task 12: Multilingual offensive language identification over social media. In Proceedings of the International Workshop on Semantic Evaluation (SemEval).

Mike Schuster and Kaisuke Nakajima. 2012. Japanese and Korean voice search. In Proceedings of the International Conference on Acoustics, Speech and Signal Processing (ICASSP).

Gudbjartur Ingi Sigurbergsson and Leon Derczynski. 2020. Offensive language and hate speech detection for Danish. In Proceedings of the 12th Language Resources and Evaluation Conference (LREC).

Abhishek Singh and Surya Pratap Singh Parmar. 2020. Voice@ SRIB at SemEval-2020 Task [9,12]: Sentiment and offensiveness detection in social media. In Proceedings of the International Workshop on Semantic Evaluation (SemEval).

Rajalakshmi Sivanaiah, Angel Deborah S, S Milton Rajendram, and Mirnalinee T T. 2020. TECHSSN at SemEval-2020 Task 12: Offensive language detection using BERT embeddings. In Proceedings of the International Workshop on Semantic Evaluation (SemEval).

Kasper Socha. 2020. KS@LTH at SemEval-2020 Task 12: Fine-tuning multi- and monolingual transformer models for offensive language detection. In Proceedings of the International Workshop on Semantic Evaluation (SemEval).

Sajad Sotudeh, Tong Xiang, Hao-Ren Yao, Sean MacAvaney, Eugene Yang, Nazli Goharian, and Ophir Frieder. 2020. GUIR at SemEval-2020 Task 12: Domain-tuned contextualized models for offensive language detection. In Proceedings of the International Workshop on Semantic Evaluation (SemEval).

Leon Strømberg-Derczynski, Rebekah Baglini, Morten H Christiansen, Manuel R Ciosici, Jacob Aarup Dalsgaard, Riccardo Fusaroli, Peter Juel Henrichsen, Rasmus Hvingelby, Andreas Kirkedal, Alex Speed Kjeldsen, et al. 2020. The Danish Gigaword Project. arXiv preprint arXiv:2005.03521.

Julia Maria Struß, Melanie Siegel, Josep Ruppenhofer, Michael Wiegand, and Manfred Klenner. 2019. Overview of GermEval task 2, 2019 shared task on the identification of offensive language. In Proceedings of KONVENS.

Yu Sun, Shuohuan Wang, Yukun Li, Shikun Feng, Hao Tian, Hua Wu, and Haifeng Wang. 2020. Ernie 2.0: A continual pre-training framework for language understanding. In Proceedings of the 34th AAAI Conference on Artificial Intelligence (AAAI).

Shardul Suryawanshi, Mihael Arcan, and Paul Buitelaar. 2020. NUIG at SemEval-2020 Task 12: Pseudo labelling for offensive content classification. In Proceedings of the International Workshop on Semantic Evaluation (SemEval).

Mircea-Adrian Tanase, Dumitru-Clementin Cercel, and Costin-Gabriel Chiru. 2020. UPB at SemEval-2020 Task 12: Multilingual offensive language detection on social media by fine-tuning a variety of BERT-based models. In Proceedings of the International Workshop on Semantic Evaluation (SemEval).

Saja Khaled Tawalbeh, Mahmoud Hammad, and Mohammad AL-Smadi. 2020. KEIS@JUST at SemEval-2020 Task 12: Identifying multilingual offensive tweets using weighted ensemble and fine-tuned BERT. In Proceedings of the International Workshop on Semantic Evaluation (SemEval).

Peter D Turney and Michael L Littman. 2003. Measuring praise and criticism: Inference of semantic orientation from association. ACM Transactions on Information Systems (TOIS), 21(4):315-346.

Moshe Uzan and HaCohen-Kerner Yaakov. 2020. JCT at SemEval-2020 Task 12: Offensive language detection in tweets using preprocessing methods, character and word n-grams. In Proceedings of the International Workshop on Semantic Evaluation (SemEval).

Ashish Vaswani, Noam Shazeer, Niki Parmar, Jakob Uszkoreit, Llion Jones, Aidan N Gomez, Łukasz Kaiser, and Illia Polosukhin. 2017. Attention is all you need. In Advances in neural information processing systems (NIPS).

Bertie Vidgen and Leon Derczynski. 2020. Directions in abusive language training data: Garbage in, garbage out. arXiv preprint arXiv:2004.01670.

Susan Wang and Zita Marinho. 2020. Nova-Wang at SemEval-2020 Task 12: OffensEmblert: an ensemble of offensive language classifiers. In Proceedings of the International Workshop on Semantic Evaluation (SemEval).

Shuohuan Wang, Jiaxiang Liu, Xuan Ouyang, and Yu Sun. 2020. Galileo at SemEval-2020 Task 12: Multilingual learning for offensive language identification using pre-trained language models. In Proceedings of the International Workshop on Semantic Evaluation (SemEval).

Zeerak Waseem, Thomas Davidson, Dana Warmsley, and Ingmar Weber. 2017. Understanding abuse: A typology of abusive language detection subtasks. In Proceedings of the First Workshop on Abusive Language Online. 
Chen Weilong, Wang Peng, Li Jipeng, Zheng Yuanshuai, Wang Yan, and Zhang Yanru. 2020. Ferryman at SemEval-2020 Task 12: BERT-based model with advanced improvement methods for multilingual offensive language identification. In Proceedings of the International Workshop on Semantic Evaluation (SemEval).

Gregor Wiedemann, Seid Yimam, and Chris Biemann. 2020. UHH-LT at SemEval-2020 Task 12: Fine-tuning of pre-trained transformer networks for offensive language detection. In Proceedings of the International Workshop on Semantic Evaluation (SemEval).

Michael Wiegand, Melanie Siegel, and Josef Ruppenhofer. 2018. Overview of the GermEval 2018 shared task on the identification of offensive language. In Proceedings of the GermEval 2018 Workshop (GermEval).

Ellery Wulczyn, Nithum Thain, and Lucas Dixon. 2017. Ex machina: Personal attacks seen at scale. In Proceedings of the 26th International Conference on World Wide Web (WWW).

Yinnan Yao, Nan Su, and Kun Ma. 2020. UJNLP at SemEval-2020 Task 12: Detecting offensive language using bidirectional transformers. In Proceedings of the International Workshop on Semantic Evaluation (SemEval).

Marcos Zampieri, Shervin Malmasi, Preslav Nakov, Sara Rosenthal, Noura Farra, and Ritesh Kumar. 2019a. Predicting the type and target of offensive posts in social media. In Proceedings of the Annual Conference of the North American Chapter of the Association for Computational Linguistics: Human Language Technology (NAACL-HLT).

Marcos Zampieri, Shervin Malmasi, Preslav Nakov, Sara Rosenthal, Noura Farra, and Ritesh Kumar. 2019 b. SemEval-2019 Task 6: Identifying and categorizing offensive language in social media (OffensEval). In Proceedings of the 13th International Workshop on Semantic Evaluation (SemEval).

Victoria Pachón Álvarez, Jacinto Mata Vázquez, José Manuel López Betanzos, and José Luis Arjona Fernández. 2020. I2C in SemEval2020 Task 12: Simple but effective approaches to offensive speech detection in Twitter. In Proceedings of the International Workshop on Semantic Evaluation (SemEval).

Anıl Özdemir and Reyyan Yeniterzi. 2020. SU-NLP at SemEval-2020 Task 12: Offensive language identification in Turkish tweets. In Proceedings of the International Workshop on Semantic Evaluation (SemEval). 


\section{A Best-Performing Teams}

Below we present a short overview of the top-3 systems for all subtasks and for all languages:

Galileo (EN B:1, EN C:1, TR A:1; DK A:2, GR A:2; AR A:3, EN A:3) This team was ranked 3rd, 1st, and 1st on the English subtasks A, B, and C, respectively; it was also ranked 1st for Turkish, 2nd for Greek and 3rd for Arabic and Danish. This is the only team ranked among the top-3 across all languages. For subtask A (all languages), they used multi-lingual pre-trained Transformers based on XLM-RoBERTa, followed by multi-lingual fine-tuning using the OffensEval data. Ultimately, they submitted an ensemble that combined XLM-RoBERTa-base and XLM-RoBERTa-large. For the English subtasks B and C, they used knowledge distillation in a teacher-student framework, using Transformers such as ALBERT and ERNIE 2.0 (Sun et al., 2020) as teacher models.

UHH-LT (EN A:1) This team was ranked 1st on the English subtask A. They fine-tuned different Transformer models on the OLID training data, and then combined them into an ensemble. They experimented with BERT-base and BERT-large (uncased), RoBERTa-base and RoBERTa-large, XLM-RoBERTa, and four different ALBERT models (large-v1, large-v2, xxlarge-v1, and xxlarge-v2). In their official submission, they used an ensemble combining different ALBERT models. They did not use the labels of the SOLID dataset, but found the tweets it contained nevertheless useful for unsupervised fine-tuning (i.e., using the MLM objective) of the pre-trained Transformers.

LT@Helsinki (DK A:1; EN C:2) This team was ranked 1st for Danish and 2nd for English subtask C. For Danish, they used NordicBERT, which is customized to Danish, and avoids some of the pre-processing noise and ambiguity introduced by other popular BERT implementations. The team further reduced orthographic lengthening to maximum two repeated characters, converted emojis to sentiment scores, and used co-occurrences of hashtags and references to usernames. They tuned the hyper-parameters of their model using 10-fold cross validation. For English subtask C, they used a very simple approach: over-sample the training data to overcome the class imbalance, and then fine-tune BERT-base-uncased.

NLPDove (GR A:1; DK A:3) This team was ranked 1st for Greek and 3rd for Danish. This team used extensive preprocessing and two data augmentation strategies: using additional semi-supervised labels from SOLID with different thresholds, and cross-lingual transfer with data selection. They further proposed and used a new metric, Translation Embedding Distance, in order to measure the transferability of instances for cross-lingual data selection. Moreover, they used data from different languages to finetune an mBERT model. Ultimately, they used a majority vote ensemble of several mBERT models, with minor variations in the parameters.

ALAMIHamza (AR A:1) This team was ranked 1st for Arabic. They used BERT to encode Arabic tweets, followed by a sigmoid classifier. They further performed translation of the meaning of emojis.

PGSG (EN B:2) The team was ranked 2nd on the English subtask B. They first fine-tuned the BERTLarge, Uncased (Whole Word Masking) checkpoint using the tweets from SOLID, but ignoring their labels. For this, they optimized for the MLM objective only, without the Next Sentence Prediction loss in BERT. Then, they built a BERT-LSTM model using this fine-tuned BERT, and adding LSTM layers on top of it, together with the [CLS] token. Finally, they used this architecture to train a Noisy Student model using the SOLID data.

ALT (AR A:2) The team was ranked 2nd for Arabic. They used an ensemble of SVM, CNN-BiLSTM and Multilingual BERT. The SVMs used character $n$-grams, word $n$-grams, word embeddings as features, while the CNN-BiLSTM learned character embeddings and further used pre-trained word embeddings as input.

SU-NLP (TR A:2) The team was ranked 2nd for Turkish. They used an ensemble of three different models: CNN-LSTM, BiLSTM-Attention, and BERT. They further used word embeddings, pre-trained on tweets, and BERTurk, BERT model for Turkish. 
Rouges (EN A:3) The team was ranked 3rd for the English subtask A. They used XLM-RoBERTa finetuned sequentially on all languages in a particular order: English, then Turkish, then Greek, then Arabic, then Danish.

NTU_NLP (EN B:3) This team was ranked 3rd on the English subtask B. They proposed a hierarchical multi-task learning approach that solves subtasks A, B, and C simultaneously, following the hierarchical structure of the annotation schema of the OLID dataset. Their architecture has three layers. The input of the first layer is the output of BERT, and its output (D1-OUT) is directly connected to the output layer for subtask A. The second layer's input is the BERT output concatenated with D1-OUT, and its output (D2-OUT) is directly connected to the output layer for subtask B. The third layer's input is the BERT output concatenated with D2-OUT, and its output is directly connected to the output layer for subtask C.

PRHLT-UPV (EN C:3) The team was ranked 3rd on the English subtask C. They used a combination of BERT and hand-crafted features, which were concatenated to the [CLS] representation from BERT. The features include the length of the tweets, the number of misspelled words, and the use of punctuation marks, emoticons, and noun phrases.

KS@LTH (GR: A: 3) This team was ranked 3rd for Greek. They experimented with monolingual and cross-lingual models, BERT and XLM-RoBERTa model, respectively, and they found BERT to perform slightly better.

KUISAIL (TR: A: 3 ) This team was ranked 3rd for Turkish. They combined BERTurk with a CNN, in a BERT-CNN model. 


\section{B Participants}

\begin{tabular}{|c|c|c|c|}
\hline Team & System Description Paper & Team & System Description Paper \\
\hline AdelaideCyC & (Herath et al., 2020) & LISAC FSDM-USMBA & (Alami et al., 2020) \\
\hline AlexU-BackTranslation-TL & (Ibrahim et al., 2020) & LT@Helsinki & (Pàmies et al., 2020) \\
\hline ALT & (Hassan et al., 2020) & NAYEL & (Nayel, 2020) \\
\hline amsqr & (Mosquera, 2020) & NLP_Passau & (Hussein et al., 2020) \\
\hline ANDES & (Arango et al., 2020) & NLPDove & (Ahn et al., 2020) \\
\hline BhamNLP & (Alharbi and Lee, 2020) & nlpUP & (Hamdy et al., 2020) \\
\hline JCT & (Uzan and Yaakov, 2020) & Nova-Wang & (Wang and Marinho, 2020) \\
\hline BRUMS & (Ranasinghe and Hettiarachchi, 2020) & NTU_NLP & (Chen et al., 2020) \\
\hline CoLi@UdS & (Chapman et al., 2020) & NUIG & (Suryawanshi et al., 2020) \\
\hline CyberTronics & (Sayanta et al., 2020) & Oulu & (Jahan and Oussalah, 2020) \\
\hline DoTheMath & (Orabe et al., 2020) & PGSG & (Pham-Hong and Chokshi, 2020) \\
\hline Duluth & (Pedersen, 2020) & pin_cod_ & (Arslan, 2020) \\
\hline FBK-DH & (Casula et al., 2020) & PRHLT-UPV & (De la Peña Sarracén and Rosso, 2020) \\
\hline Ferryman & (Weilong et al., 2020) & problemConquero & (Laud et al., 2020) \\
\hline Galileo & (Wang et al., 2020) & PUM & (Janiszewski et al., 2020) \\
\hline Garain & (Garain, 2020) & Rouges & (Dadu and Pant, 2020) \\
\hline GruPaTo & (Colla et al., 2020) & SalamNET & (Husain et al., 2020) \\
\hline GUIR & (Sotudeh et al., 2020) & SINAI & (Plaza-del Arco et al., 2020) \\
\hline Hitachi & (Ravikiran et al., 2020) & Smatgrisene & (Henrichsen and Rathje, 2020) \\
\hline $\mathrm{I} 2 \mathrm{C}$ & (Álvarez et al., 2020) & Sonal.kumari & (Kumari, 2020) \\
\hline iCompass & (Messaoudi et al., 2020) & SRIB2020 & (Singh and Parmar, 2020) \\
\hline IIITG-ADBU & (Baruah et al., 2020) & SSN_NLP_MLRG & (Kalaivani and D., 2020) \\
\hline IITP-AINLPML & (Ghosh et al., 2020) & SU-NLP & (Özdemir and Yeniterzi, 2020) \\
\hline INGEOTEC & (Miranda-Jiménez et al., 2020) & TAC & (Anwar and Baig, 2020) \\
\hline IR3218-UI & (Kurniawan et al., 2020) & TECHSSN & (Sivanaiah et al., 2020) \\
\hline IRlab@IITV & (Saroj et al., 2020) & TheNorth & (Alonso et al., 2020) \\
\hline IRLab_DAIICT & (Parikh et al., 2020) & UHH-LT & (Wiedemann et al., 2020) \\
\hline KAFK & (Das et al., 2020) & UJNLP & (Yao et al., 2020) \\
\hline KDELAB & (Hanahata and Aono, 2020) & UNT & (Fromknecht and Palmer, 2020) \\
\hline KEIS@JUST & (Tawalbeh et al., 2020) & UoB & (Lim and Madabushi, 2020) \\
\hline KS@LTH & (Socha, 2020) & UPB & (Tanase et al., 2020) \\
\hline KUISAIL & (Safaya et al., 2020) & UTFPR & (Boriola and Paetzold, 2020) \\
\hline Kungfupanda & (Dai et al., 2020) & WOLI & (Otiefy et al., 2020) \\
\hline Lee & (Junyi et al., 2020) & $\mathrm{XD}$ & (Dong and Choi, 2020) \\
\hline LIIR & (Ghadery and Moens, 2020) & YNU_oxz & (Ou et al., 2020) \\
\hline
\end{tabular}

Table 12: The teams that participated in OffensEval-2020 and submitted system description papers and the corresponding reference to their papers. 


\begin{tabular}{|c|c|c|c|c|c|c|c|}
\hline Team & A-Arabic & A-Danish & A-Greek & A-Turkish & A-English & B-English & C-English \\
\hline AdelaideCyC & & & & & & $\checkmark$ & \\
\hline AlexU-BackTranslation-TL & & & & & $\checkmark$ & $\checkmark$ & $\checkmark$ \\
\hline ALT & $\checkmark$ & & & & $\checkmark$ & & \\
\hline alaeddin & & & & $\checkmark$ & & & \\
\hline ALAMIHamza & $\checkmark$ & & & & & & \\
\hline $\begin{array}{l}\text { AMR-KELEG } \\
\text { amsqr }\end{array}$ & $\checkmark$ & & & & $\checkmark$ & & \\
\hline ANDES & $\checkmark$ & $\checkmark$ & $\checkmark$ & $\checkmark$ & $\checkmark$ & & \\
\hline ARA & & $\checkmark$ & $\checkmark$ & $\checkmark$ & $\checkmark$ & & \\
\hline asking28 & $\checkmark$ & & & & & & \\
\hline Better Place & & & & & $\checkmark$ & & \\
\hline BhamNLP & $\checkmark$ & & & & & & \\
\hline Bodensee & & & & & $\checkmark$ & $\checkmark$ & $\checkmark$ \\
\hline Bushr & $\checkmark$ & & & & & & \\
\hline byteam & & & & & $\checkmark$ & & \\
\hline Coffee_Latte & & & & & $\checkmark$ & & \\
\hline CoLi@UdS & $\checkmark$ & & $\checkmark$ & $\checkmark$ & $\checkmark$ & $\checkmark$ & \\
\hline COMA & $\checkmark$ & & & & & & \\
\hline $\begin{array}{l}\text { CyberTronics } \\
\text { doxaAI }\end{array}$ & $\checkmark$ & & $\checkmark$ & $\checkmark$ & $\checkmark$ & & \\
\hline Duluth & & & & & $\checkmark$ & $\checkmark$ & $\checkmark$ \\
\hline erfan & $\checkmark$ & & & & $\checkmark$ & & \\
\hline f_shahaby & & & & $\checkmark$ & & & \\
\hline fatemah & $\checkmark$ & & $\checkmark$ & $\checkmark$ & & & \\
\hline FBK-DH & $\checkmark$ & $\checkmark$ & $\checkmark$ & $\checkmark$ & $\checkmark$ & & \\
\hline FERMI & & $\checkmark$ & $\checkmark$ & $\checkmark$ & $\checkmark$ & $\checkmark$ & $\checkmark$ \\
\hline Ferryman & 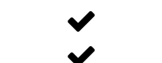 & $\checkmark$ & $\checkmark$ & $\checkmark$ & $\checkmark$ & $\checkmark$ & $\checkmark$ \\
\hline $\begin{array}{l}\text { frankakorpel } \\
\text { Galileo }\end{array}$ & $\checkmark$ & $\checkmark$ & $\checkmark$ & $\checkmark$ & $\checkmark$ & $\checkmark$ & $\checkmark$ \\
\hline GruPaTo & & $\checkmark$ & & $\checkmark$ & $\checkmark$ & 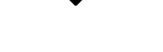 & \\
\hline GUIR & & & & & $\checkmark$ & $\checkmark$ & $\checkmark$ \\
\hline hamadanayel & $\checkmark$ & & & & & & \\
\hline HateLab & & & & & $\checkmark$ & $\checkmark$ & $\checkmark$ \\
\hline hhaddad & $\checkmark$ & & & & & & \\
\hline Hitachi & & & & & $\checkmark$ & & \\
\hline $\mathrm{I} 2 \mathrm{C}$ & $\checkmark$ & & & $\checkmark$ & $\checkmark$ & $\checkmark$ & \\
\hline iaf7 & $\checkmark$ & & & & & & \\
\hline IASBS & & & & $\checkmark$ & $\checkmark$ & & \\
\hline IIITG-ADBU & & & & & $\checkmark$ & $\checkmark$ & $\checkmark$ \\
\hline IITP-AINLPML & & & & & $\checkmark$ & $\checkmark$ & $\checkmark$ \\
\hline IJS & & $\checkmark$ & $\checkmark$ & $\checkmark$ & $\checkmark$ & $\checkmark$ & $\checkmark$ \\
\hline INGEOTEC & $\checkmark$ & $\checkmark$ & $\checkmark$ & $\checkmark$ & $\checkmark$ & $\checkmark$ & $\checkmark$ \\
\hline IR3218-UI & & $\checkmark$ & & & $\checkmark$ & & \\
\hline IRlab@IITV & & & $\checkmark$ & & $\checkmark$ & $\checkmark$ & \\
\hline IRLab_DAIICT & & & & & $\checkmark$ & $\checkmark$ & \\
\hline IS & & & & & & & $\checkmark$ \\
\hline ITNLP & & & & & $\checkmark$ & & $\checkmark$ \\
\hline IU-UM@LING & & $\checkmark$ & $\checkmark$ & $\checkmark$ & $\checkmark$ & $\checkmark$ & $\checkmark$ \\
\hline IUST & & $\checkmark$ & $\checkmark$ & $\checkmark$ & $\checkmark$ & & \\
\hline JAK & & $\checkmark$ & $\checkmark$ & $\checkmark$ & & & \\
\hline janecek1 & & & & & $\checkmark$ & & \\
\hline jbern & $\checkmark$ & & & & & & \\
\hline JCT & $\checkmark$ & $\checkmark$ & $\checkmark$ & $\checkmark$ & & & \\
\hline $\begin{array}{l}\text { jlee24282 } \\
\text { jooyeon Lee }\end{array}$ & $\checkmark$ & & & $\checkmark$ & & & \\
\hline KAFK & & & & & $\checkmark$ & $\checkmark$ & $\checkmark$ \\
\hline KarthikaS & & & & & $\checkmark$ & $\checkmark$ & \\
\hline KDELAB & & & & & $\checkmark$ & $\checkmark$ & $\checkmark$ \\
\hline KEIS@JUST & & $\checkmark$ & $\checkmark$ & $\checkmark$ & & $\checkmark$ & $\checkmark$ \\
\hline
\end{tabular}

Table 13: Overview of team participation in the subtasks (part 1). 


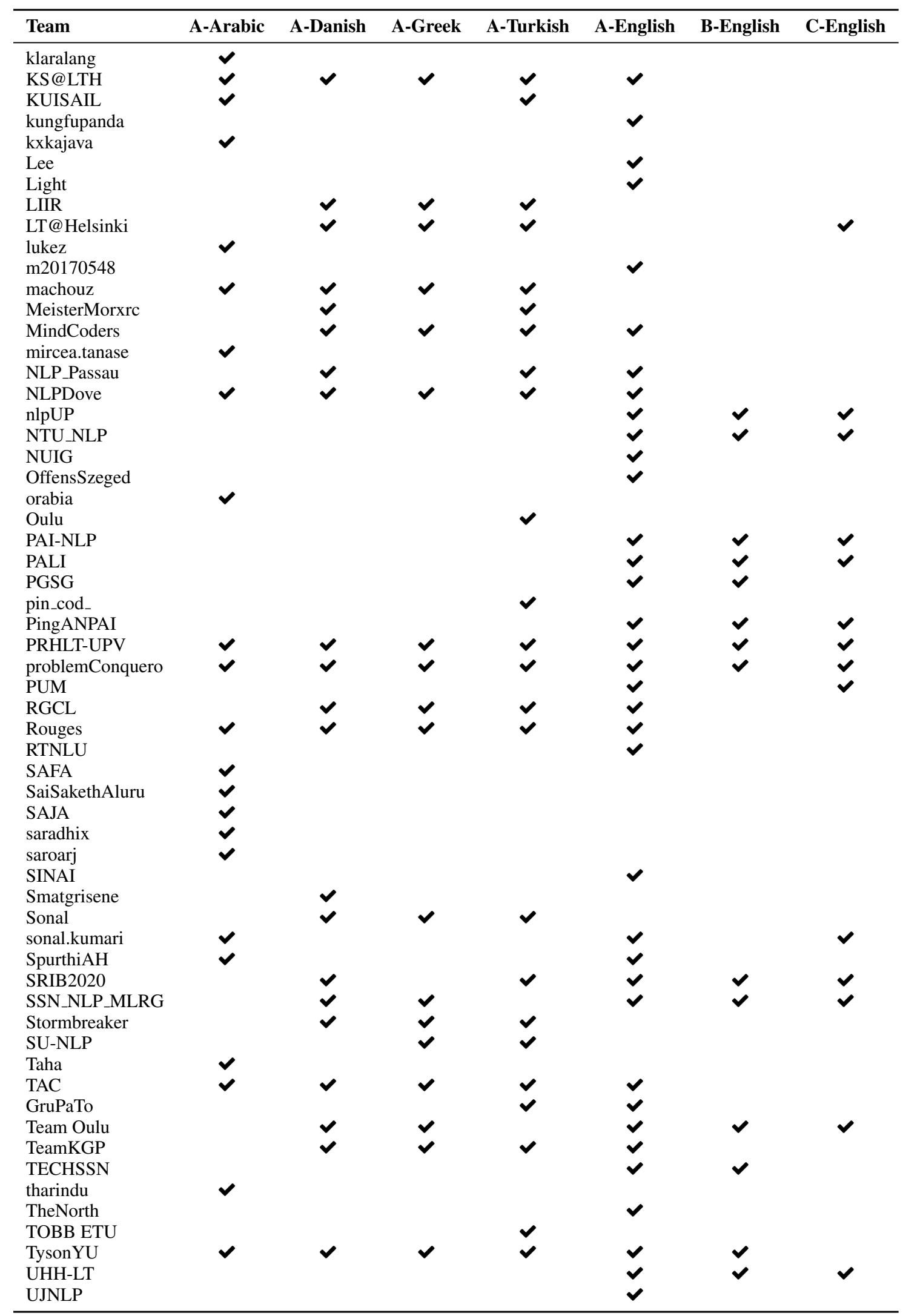

Table 14: Overview of team participation in the subtasks (part 2). 


\begin{tabular}{lccccccc}
\hline Team & A-Arabic & A-Danish & A-Greek & A-Turkish & A-English & B-English & C-English \\
\hline ultraviolet & & & & & & & $\checkmark$ \\
UNT Linguistics & & & & $\checkmark$ & $\checkmark$ & \\
UoB & & & & & $\checkmark$ & $\checkmark$ & \\
UTFPR & & & & & $\checkmark$ & $\checkmark$ & $\checkmark$ \\
VerifiedXiaoPAI & & & & & $\checkmark$ & & \\
wac81 & $\checkmark$ & $\checkmark$ & $\checkmark$ & $\checkmark$ & & $\checkmark$ & \\
will_go & & $\checkmark$ & $\checkmark$ & & $\checkmark$ & & \\
KUISAIL & & & & & $\checkmark$ & & \\
Wu427 & & & & & \\
XD & & & & $\checkmark$ & & \\
yasserotiefy & $\checkmark$ & & & & & \\
YNU_2016 & $\checkmark$ & & & & & \\
zahra.raj & $\checkmark$ & & & & & \\
zoher_orabe & $\checkmark$ & & & &
\end{tabular}

Table 15: Overview of team participation in the subtasks (part 3). 